\title{
IL-4 Modulates Macrophage Polarization in Ankylosing Spondylitis
}

\author{
Shishui Lin ${ }^{\mathrm{a} b}$ Meiguang Qiu ${ }^{\mathrm{b}}$ Jianting Chen ${ }^{\mathrm{a}}$ \\ aDepartment of Spinal Surgery, Nanfang Hospital, Southern Medical University, Guangzhou,

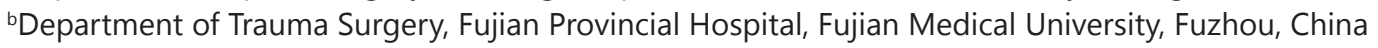

\section{Key Words}

Osteoclast (OC) • Interleukin 4 (IL-4) • Receptor activator of NF-kB ligand (RANKL) • Macrophage polarization $\cdot$ Histone deacetylation

\begin{abstract}
Background/Aims: Osteoclasts (OC) originate from monocytes/macrophages and play a critical role in the development of ankylosing spondylitis (AS). Receptor activator of NF$\mathrm{kB}$ ligand (RANKL) is critical for the differentiation and maturation of OC from monocytes/ macrophages, the underlying mechanisms of which processes have not been completely elucidated. Interleukin 4 (IL-4) attenuates the pathogenesis of AS via ill-defined mechanisms. Methods: We used a proteoglycan-induced arthritis (PGIA) model in Balb/c mice to study AS in humans. We injected IL-4 into the articular cavity and evaluated its effects on PGIA by incidence of arthritis, clinical and pathological arthritis severity and PET tracer uptake. We isolated and analyzed the number and polarization of macrophages in the articular cavity before and after IL-4 treatment. We analyzed RANKL levels in macrophage subtypes. We then isolated bone-marrow derived macrophages and treated them with IL-4 in vitro, with or without histone deacetylase inhibitors trichostatin A (TSA), and then analyzed the polarization of cultured macrophages before and after IL-4 treatment and RANKL levels in macrophage subtypes. Results: IL-4 treatment decreased the incidence and severity of arthritis in a mouse AS model, and polarized macrophages from a classical M1 subtype to an M2 subtype in vivo and in vitro. RANKL was predominantly produced by $M 1$, but not by $M 2$ macrophages. IL-4mediated inhibition of RANKL in macrophages was abolished by TSA. Conclusion: Our data suggest that the therapeutic effects of IL-4 on AS may result from a M1-to-M2 macrophage polarization and its inhibition of RANKL expression on macrophages, possibly through enhanced histone deacetylation.

Jianting Chen

KARGER 125
Department of Spinal Surgery, Nanfang Hospital, Southern Medical University, 1838 North Guangzhou Avenue, Guangzhou 510515 (China)

Tel.+862061641114, E-Mail jianting_chen@163.com 


\section{Cellular Physiology Cell Physiol Biochem 2015;35:2213-2222 \begin{tabular}{ll|l} 
DOI: 10.1159/000374026 & $\begin{array}{l}\text { O 2015 S. Karger AG, Basel } \\
\text { www.karger.com/cpb }\end{array}$ \\
\hline
\end{tabular} \\ Lin/Qiu/Chen: IL-4 Modulates Macrophages in AS}

\section{Introduction}

Spondyloarthritis (SpA) includes reactive arthritis, psoriatic arthritis, colitic arthropathies, undifferentiated SpA and ankylosing spondylitis (AS), the latter of which is characterized by chronic inflammation of the sacroiliac peripheral joints and the absence of rheumatoid factor [1-4]. AS often leads to significant spinal disease and peripheral arthritis, which can manifest as chronic back pain and a progressive spinal ankyloses [1-4]. The disease strikes predominantly men between the ages of 20 and 40 years [1-4].

The pathogenesis of AS has been found to be strongly related to major histocompatibility complex (MHC) class I since a majority of AS patients have human leukocyte antigen (HLA)-B27 (HLA-B27) gene [1-4]. Although the mechanisms of AS onset remain incompletely elucidated, the involvement of osteoclasts (OCs) and a set of pro-inflammatory cytokines (e.g. TNF- $\alpha$, IL-6, and IL-10) have been discerned [1-4].

A set of new bone formation and low bone mass and density occur in AS patients, suggesting the involvement of OCs and an imbalance of receptor activator of NF- $\kappa B$ ligand (RANKL) system [5-7]. OCs are present only in bone, and multinucleated giant cells with the capacity to resorb mineralized tissues [5-7]. OCs are derived from hematopoietic progenitors of the monocyte-macrophage lineage [5-10]. Bone-marrow derived macrophages are believed to be the major precursor cells for OCs [5-10]. Osteoblasts and stromal stem cells bind to OCs through RANKL, which induces expression of RANK surface of osteoclasts and their precursors [5, 8-10]. Osteoprotegerin (OPG) is also secreted by osteoblasts and stromal stem cells and protects the skeleton from excessive bone resorption by binding to RANKL and preventing it from interacting with RANK [5-10]. This RANKL/RANK/OPG system was discovered in the mid-1990s [11-13]. The RANKL/OPG ratio in bone marrow is thus an important determinant of bone mass in normal and disease states [5-10]. Increased RANKL levels have been suggested to enhance the disease progress of AS [14].

Aside from the classically activated macrophages (also called M1 macrophages), which respond to type I helper T cells (Th1) and generate reactive oxygen species and nitric oxide to kill pathogens and cells, there are also alternatively activated macrophages (M2 macrophages), which respond to type II helper T cells (Th2) to mediate humoral immunity and tissue repair [15-19]. The adaption of macrophage phenotypes is called polarization.

Interleukin 4 (IL-4) is a pleiotropic cytokine that plays an essential role in $\mathrm{T}$ and $\mathrm{B}$ lymphocyte differentiation to initiate type 2 immune responses [20-25]. In vivo treatment with IL-4 was effective in various autoimmune models, including collagen-induced arthritis [21-25] and proteoglycan-induced arthritis (PGIA) [20], mouse models for AS. This is due to the fact that IL-4 acts as an anti-inflammatory cytokine that modulates macrophage polarization and activity through suppression of Th1-mediated pro-inflammatory effects and enhancement of Th2-mediate anti-inflammatory effects [26-28], possibly via modulation of histone deacetylation [29-33]. M2 macrophages are typically found in Th2-dominated responses, as the Th2-driving IL-4 is a strong inducer of M2 polarization [15-19]. However, the molecular mechanisms underlying the therapeutic effects of IL-4 on AS are not defined. Moreover, macrophage polarization in AS, especially by IL-4 treatment, has not been studied.

Here, we injected IL-4 into the articular cavity of Balb/c mice and evaluated its effects on PGIA. We isolated and analyzed the number and polarization of macrophages in the articular cavity before and after IL-4 treatment, as well as the RNAKL levels in macrophage subtypes. We isolated bone-marrow derived macrophages and treated them with IL-4 in vitro, with and without histone deacetylase inhibitors trichostatin A (TSA), and then performed similar analyses in vivo. We found that IL-4 treatment inhibited the incidence and severity of arthritis in a mouse AS model, and polarized macrophages from a classical M1 subtype to a M2 subtype in vivo and in vitro. RANKL was predominantly produced by M1, but not by M2 macrophages. IL-4-mediated inhibition of RANKL in macrophages was abolished by TSA. 


\section{Materials and Methods}

\section{Mouse handling}

All mouse experiments were approved by and performed according to the guidelines of the IACUC of Nanfang Hospital of Southern Medical University. Only 12-week-old female Balb/c mice (Jackson lab, Bar Harbor, ME, USA) were used for in vivo experiments. The mice were kept in specific pathogen free (SPF) conditions.

\section{Proteoglycan-induced arthritis (PGIA) model}

Female Balb/c mice were i.p. injected with $100 \mu \mathrm{g}$ of cartilage proteoglycans (Sigma-Aldrich, St. Louis, MO, USA) on weeks 0,3 , and 6 . The first and third injections of proteoglycan were given in CFA (Difco, Detroit, MI, USA) and the second injection of proteoglycan was given in IFA (Difco) as has been previously described [20].

\section{Intra-articular injection of $I L-4$}

Intra-articular injection of IL-4 was performed as has been described before [34]. Mice were anesthetized with $2.5 \%$ isoflurane in $100 \%$ oxygen at a flow rate of $1 \mathrm{~L} /$ minute. Hair was removed from both knee joints with a hair removal cream (Veet) to facilitate direct observation. The right knee joint of the mice was punctured once with a $30 \mathrm{G}$ needle below the patella to induce a hemorrhage. Subsequently a $3 \mu \mathrm{l}$ solution, containing 100ng recombinant mouse IL-4 (R\&D systems, Los Angeles, CA, USA) or vehicle only (phosphate-buffered saline containing $20 \mathrm{mmol} / \mathrm{l}$ Tris and $100 \mathrm{mmol} / \mathrm{l} \mathrm{NaCl}, \mathrm{pH} 7.5$ ), was injected through a 33G needle in mice for each treatment. After these injections, the mice received acetaminophen via their drinking water to relieve pain until the end of the experiment.

\section{Assessment of arthritis}

Mice were scored blindly every other week for clinical signs of arthritis as has been described before [35]: 0 (no symptoms), 1 (redness and swelling in one toe), 2 (redness and swelling in more than one toe), 3 (toe stiffness), and 4 (deformity or ankle involvement). Hind paw forefeet were studied by histomorphology as has been described [35]: 0 (normal toe), 1 (acute inflammation including dactylitis), 2 (entheseal cell proliferation), 3 (cartilage formation), 4 (bone formation), and 5 (joint ankylosis). A cumulative score from all toes was calculated. The shown arthritis data represent results from one of the three experiments.

Positron Emission Tomography (PET) imaging and Dual Energy X-ray Absorptiometry (DEXA)

The imaging of the absorption of 2-[ $\left.{ }^{18} \mathrm{~F}\right]$ fluoro-2-deoxy-D-glucose $\left({ }^{18} \mathrm{~F}-\mathrm{FDG}\right)$ in mice has been described before [36]. Briefly, the mice were imaged using a Focus 220 microPET $®$ scanner (Concorde Microsystems Inc., Knoxville, TN, USA). The mice were anesthetized with $2.5 \%$ isoflurane in $100 \%$ oxygen at a flow rate of $1 \mathrm{~L} /$ minute and 7.4 MBq of ${ }^{18} \mathrm{~F}$-FDG was administered by lateral tail vein injection. One hour after injection, a static, ten minute scan was acquired. Images were reconstructed using a maximum posteriori algorithm from the vendor with 18 iterations and uniform resolution. The images were displayed in Amide's a Medical Imaging Data Examiner and three-dimensional spheroid regions of interest (ROIs) were drawn. The mean radioactivity in the ROIs was converted to percent of the injected dose per gram of tissue (\%ID/g) by a calibrated cylinder factor and after division with the injected dose (corrected for residual and decay). Standardized uptake values (SUV) were obtained by multiplying the \% ID/g of the toe area by the weight of the animal. Bone mineral content was assessed by dual X-ray absorptiometry using Piximus densitometer (Lunar, Madison, WI, USA).

Isolation and culture of bone-marrow derived mouse macrophages

Isolation of bone-marrow derived mouse macrophages has been described before [37]. Marrow from 12 female, week-old Balb/c mice was flushed out with vehicle solution (phosphate-buffered saline containing $20 \mathrm{mmol} / \mathrm{l}$ Tris and $100 \mathrm{mmol} / \mathrm{l} \mathrm{NaCl}, \mathrm{pH} 7.5$ ) through a 23G needle. Cells were pre-treated with FITC-conjugated F4/80 antibody (Becton-Dickinson Biosciences, San Jose, CA, USA) and then sorted for positive cells by flow cytometry. Purified F4/80-positive macrophages were cultured in Dulbecco's Modified Eagle Medium/F12 (DMEM/F12; Invitrogen, St. Louis, MO, USA) supplemented with $10 \mathrm{mmol} / \mathrm{l}$ L-glutamine, $100 \mathrm{U} / \mathrm{ml}$ penicillin, $100 \mu \mathrm{g} / \mathrm{ml}$ streptomycin and $100 \mathrm{U} / \mathrm{ml}$ recombinant M-CSF (mouse M-CSF, R\&D Systems). 


\section{Cellular Physiology Cell Physiol Biochem 2015;35:2213-2222 \begin{tabular}{ll|l} 
and Biochemistry & $\begin{array}{l}\text { DOI: 10.1159/000374026 } \\
\text { Published online: April 08, } 2015\end{array}$ & $\begin{array}{l}\text { O) 2015 S. Karger AG, Basel } \\
\text { www.karger.com/cpb }\end{array}$ \\
\cline { 1 - 2 }
\end{tabular}}

Lin/Qiu/Chen: IL-4 Modulates Macrophages in AS

\section{Fluorescence-activated cell sorting (FACS) for macrophage phenotype}

Cells from the articular cavity of the mice were flushed out with vehicle solution (phosphate-buffered saline containing $20 \mathrm{mmol} / \mathrm{l}$ Tris and $100 \mathrm{mmol} / \mathrm{l} \mathrm{NaCl}, \mathrm{pH} 7.5$ ) through a 23G needle. Cells from culture were detached from the culture plates with $0.25 \%$ Trypsine solution (Invitrogen), washed three times with vehicle solution, re-suspended in vehicle solution, labeled with FITC-conjugated F4/80 antibody and PEcy7-conjugated CD206 antibody (Becton-Dickinson Biosciences), and then sorted for macrophage M1 and M2 subtypes. Data were analyzed and quantified using Flowjo software (Flowjo LLC, Ashland, OR, USA).

\section{$R T-q P C R$}

RNA was extracted from purified macrophages after FACS with RNeasy (Qiagen, Hilden, Germany). Complementary DNA (cDNA) synthesis was performed by reserve transcription. Quantitative PCR (RTqPCR) was performed in duplicates with QuantiTect SYBR Green PCR Kit (Qiagen). All primers were purchased from Qiagen. Quantification used ddCT method. Values of genes were first normalized against $\beta$-actin, and then compared to controls.

\section{Statistics}

GraphPad Prism software (GraphPad Software, Inc. La Jolla, CA, USA) was used for statistic analyses. Unpaired two-tailed Student $t$ test was applied for comparison between two groups, and one-way ANOVA with the Tukey posttest was applied for comparison between three or more groups. Data were represented as mean \pm SD and were considered significant if $\mathrm{p}<0.05$.

\section{Results}

\section{IL-4 inhibited development of PGIA in mice}

Eighty Balb/c mice were immunized with proteoglycan through triple injections at week 0,3 and 6, as has been described [20]. At 10 weeks, 40 mice received Intra-articular injection of IL-4, while the other 40 received vehicle. At 10 and 11 weeks each, 20 mice $(10$ weeks: 20 mice immediately after injections of IL-4 (10)/Vehicle (10), 11 weeks: 10 mice that had received IL-4 and 10 mice that had received vehicle) were sacrificed and analyzed for macrophages in the articular cavity that had received injections of IL-4/Vehicle. The other 40 mice (20 that had received IL- 4 and 20 that had received vehicle) were monitored for development of arthritis at 8,10,12 and 14 weeks by evaluating the clinical severity and pathological severity (Fig. 1A).

We found that IL-4-treated mice exhibited a significant decrease in the incidence of arthritis (Fig. 1B), a significant decrease in clinical severity (Fig. 1C), and a significant decrease in the pathological severity (Fig. 1D), compared to vehicle-injected mice. Since the pathogenesis of AS is characterized by infiltration of neutrophils and mononuclear inflammatory cells, and since ${ }^{18} \mathrm{~F}$-FDG has been shown to accumulate in activated neutrophils and macrophages and thus serves as a marker of inflammation [38], we injected ${ }^{18} \mathrm{~F}$-FDG into IL-4-treated mice and quantified the inflammation by ${ }^{18} \mathrm{~F}-\mathrm{FDG}$ uptake using PET scan in the paws of the mice. We found that the uptake of tracer was significantly reduced in the IL-4treated mice at 14 weeks, by quantification (Fig. 1E), and on a representative image (Fig. 1F). Together, these data suggest that IL-4 treatment significantly reduced severity of PGIA. Thus, this model is validated for further mechanistic studies of AS.

IL-4 did not alter macrophage number, but induced macrophage polarization in PGIA

Since IL-4 has been identified as a macrophage polarization trigger, and the macrophage is a precursor of OC in AS, we hypothesized that IL-4 may alter macrophage polarization in PGIA. In order to examine this hypothesis, we isolated cells from the articular cavity at 11 weeks, 1 week after IL-4 injection (Fig. 1A), and analyzed and purified macrophages (based on F4/80) as well as their subtypes (M1 based on CD206-negative, M2 based on CD206-positive) by FACS (Fig. 2A). We did not find changes in the percentage of macrophages in the total cells from articular cavity (Fig. 2B), but detected a significant polarization of macrophages to an 

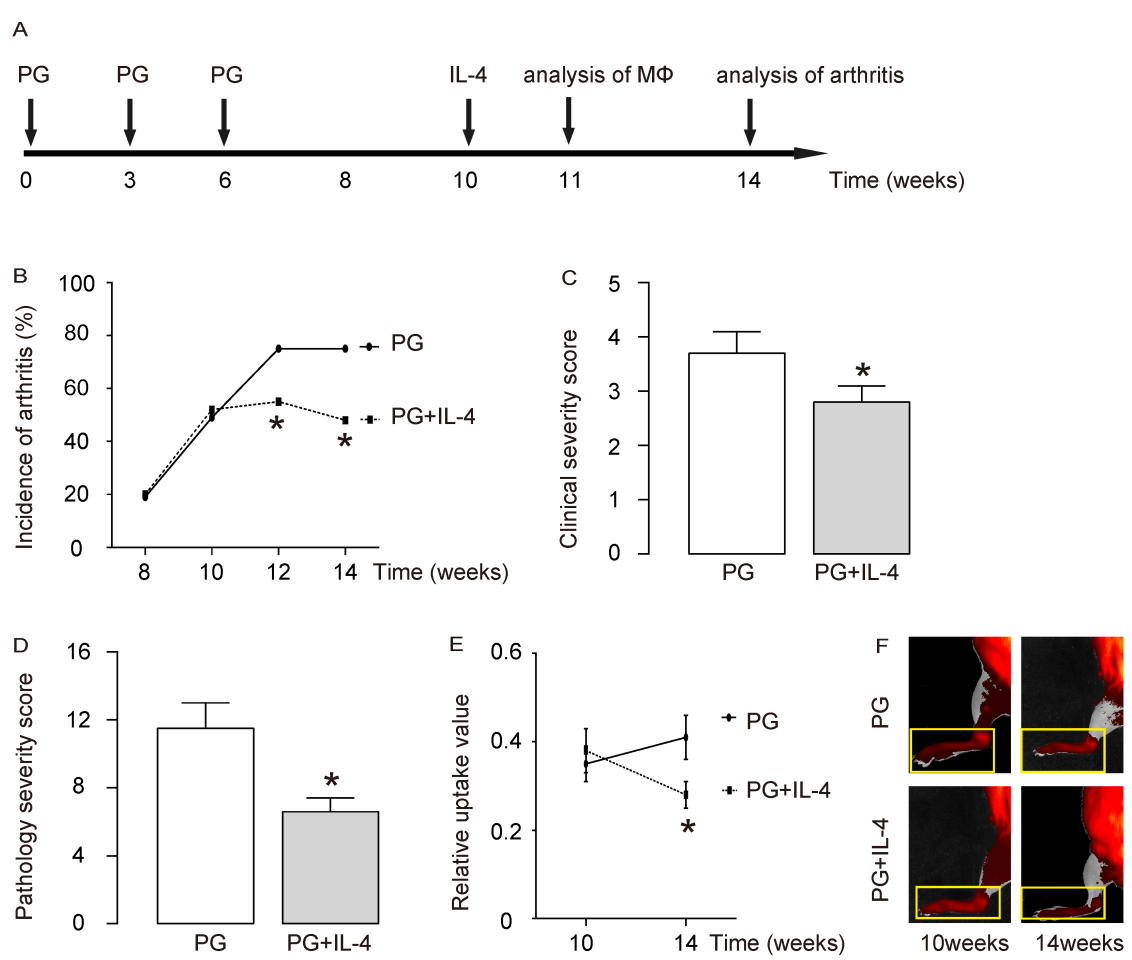

Fig. 1. IL-4 inhibited development of PGIA in mice. (A) Schematic of the model: Eighty Balb/c mice were immunized with proteoglycan through triple injections at week 0, 3 and 6. At 10 weeks, 40 mice received Intra-articular injection of IL-4, while the other 40 received vehicle. At 10 and 11 weeks each, 20 mice (10 weeks: 20 mice immediately after injections of IL-4 (10)/Vehicle (10), 11 weeks: 10 mice that had received IL-4 and 10 mice that had received vehicle) were sacrificed and analyzed for macrophages in the articular cavity that had received injections of IL-4/Vehicle. The other 40 mice 20 that had received IL- 4 and 20 that had received vehicle) were monitored for development of arthritis at 8,10,12 and 14 weeks by evaluating the clinical severity and pathological severity. (B) Incidence of arthritis. $n=20$. (C) Clinical severity score. (D) Pathological severity score. $n=20$. (E-F) ${ }^{18} \mathrm{~F}$-FDG was injected into IL-4-treated mice and the inflammation was quantified by ${ }^{18} \mathrm{~F}-\mathrm{FDG}$ uptake using PET scan in the paws of the mice, shown by quantification (E), and by representative image (F). PG: proteoglycan; MФ: macrophages; $n=20$. ${ }^{*} \mathrm{p}<0.05$. Statistics: Unpaired twotailed Student t test.

M2 subtype (Fig. 2C), by IL-4 treatment. These data confirmed our hypothesis, suggesting that IL-4 may not alter macrophage number, but may induce macrophage polarization in PGIA.

M1, but not M2 macrophages expressed high levels of RANKL

In order to find out whether macrophage polarization plays a role in the therapeutic effects of IL-4 in PGIA, we analyzed RANKL levels in isolated M1 and M2 macrophages. RANKL is a critical inducer of AS and RANKL has been shown to be critical for OC differentiation. We found that M2 macrophages had significantly lower transcript levels of RANKL, compared to M1 macrophages, regardless of the sources and experimental time points (Fig. 3). Since IL-4 induced M1-to-M2 polarization, it then resulted in significant decreases in RANKL levels, which subsequently prevented OC differentiation.

IL-4 induced M1-to-M2 macrophage polarization in vitro, possibly through enhanced histone deacetylation

To confirm these findings in vivo, we examined the effects of IL-4 on macrophages and RANKL levels in macrophages in vitro, to exclude the possibility of model-dependence on 
Fig. 2. IL-4 did not alter macrophage number, but induced macrophage polarization in PGIA. (A-C) We isolated cells from the articular cavity at 11 weeks, 1 week after IL-4 injection, and analyzed and purified macrophages (based on F4/80) as well as their subtypes (M1 based on CD206-negative, M2 based on CD206-positive) by FACS (A). (B) The percentage of macrophages in the total cells from articular cavity. (C) The polarization of macrophages was determined by $\mathrm{M} 1 / \mathrm{M} 2$ ratio. $\mathrm{n}=10$. ${ }^{*} \mathrm{p}<0.05$. NS: non-significant. Statistics: Unpaired two-tailed Student t test.

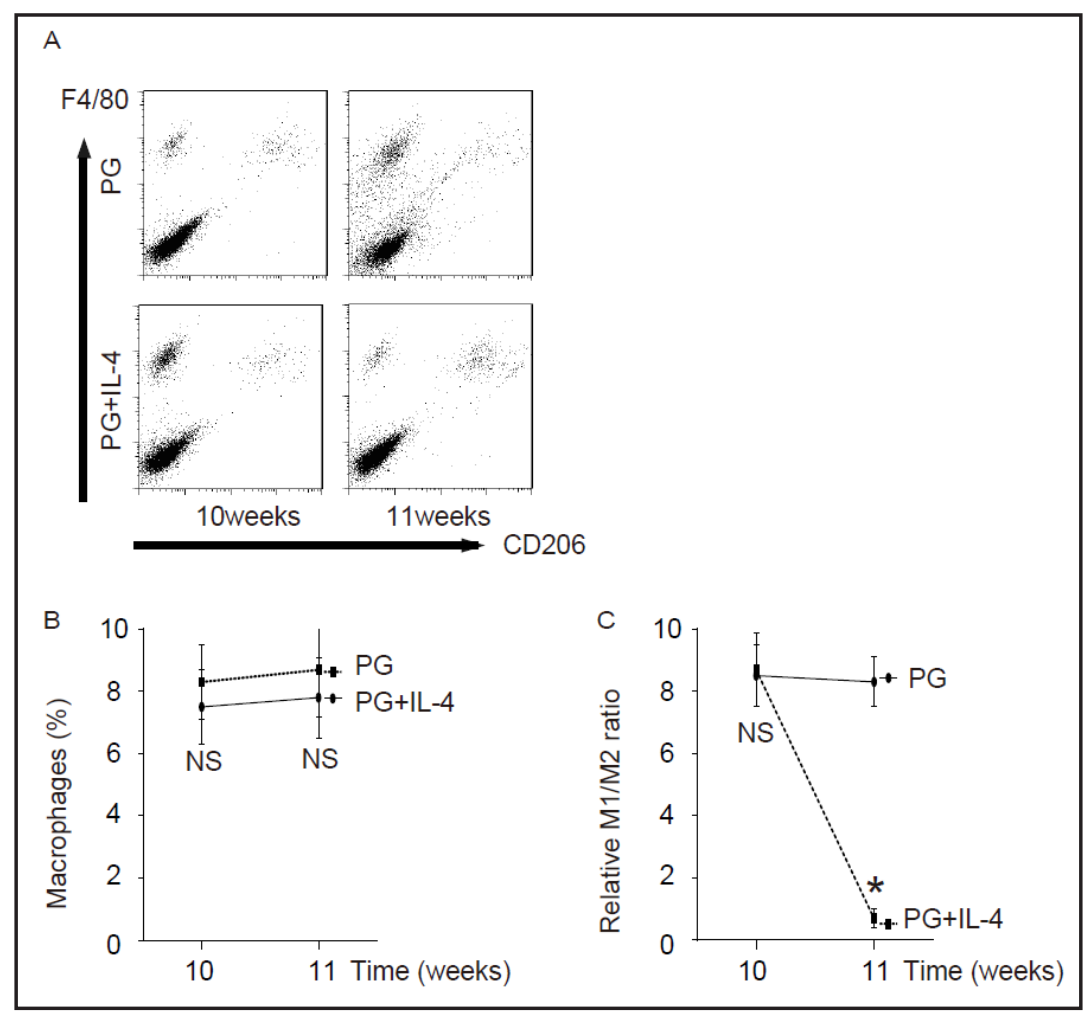

Fig. 3. M1, but not M2 macrophages expressed high levels of RANKL. RANKL transcript levels in isolated M1 and M2 macrophages were determined by RT-qPCR. $n=10 .{ }^{*} \mathrm{p}<0.05$. Statistics: Unpaired twotailed Student t test.

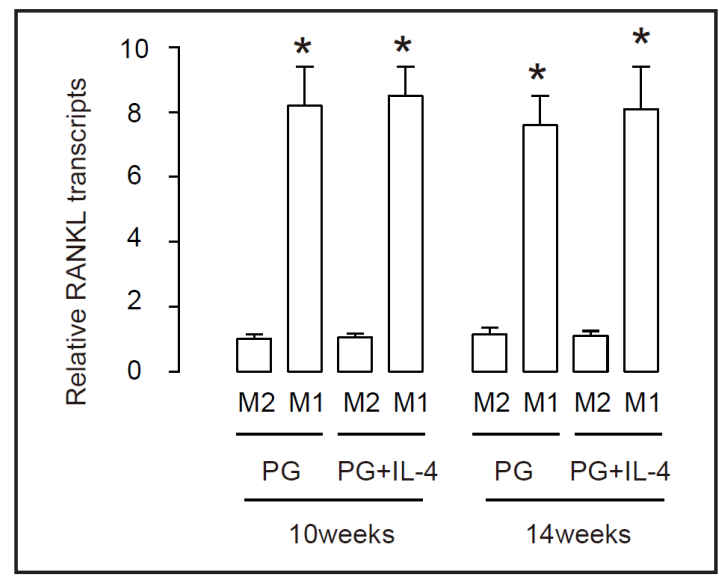

the in-vivo environment. We isolated mouse macrophages from bone marrow and then put these macrophages in culture, treated with or without IL-4 (Fig. 4A). We found that IL-4 similarly induced M1-to-M2 polarization in vitro, without the need for the PGIA environment (Fig. 4B). Moreover, M2 macrophages, regardless of the sources from different experimental conditions, expressed significantly lower RANKL transcripts, compared to M1 macrophages (Fig. 4C). These data suggest that IL-4-induces M1-to-M2 polarization, and decreases in RANKL levels in macrophages do not require a PGIA environment.

Since IL-4 has been shown to conduct its functions through induction of histone deacetylation, we thus added TSA, an inhibitor for histone deacetylation, into IL-4-treated macrophage culture (Fig. 4A). TSA significantly and nearly completely abolished the effects of IL-4-induced M1-to-M2 polarization (Fig. 4B), without affecting the RANKL levels in M1 and M2 macrophages (Fig. 4C). Together, these data suggest that IL-4 induced M1-to-M2 macrophage polarization in vitro, possibly through enhancing histone deacetylation (Fig. 5). 
Fig. 4. IL-4 induced M1-to-M2 macrophage polarization in vitro, possibly through enhanced histone deacetylation. (A) Schematic: We isolated mouse macrophages from bone marrow and then put these macrophages in culture, treated with/ without IL-4, and with/without TSA. (B) The polarization of macrophages was determined by M1/ M2 ratio. (C) RANKL transcript levels in isolated M1 and M2 macrophages were determined by RT-qPCR. $n=10 .{ }^{*} \mathrm{p}<0.05$. Statistics: one-way ANOVA with the Tukey posttest.

Fig. 5. Schematic of the model. In AS, IL-4 treatment induces M1-to-M2 macrophage polarization, possibly through enhancing histone deacetylation, which decreases RANKL levels to inhibit OC activation and differentiation.
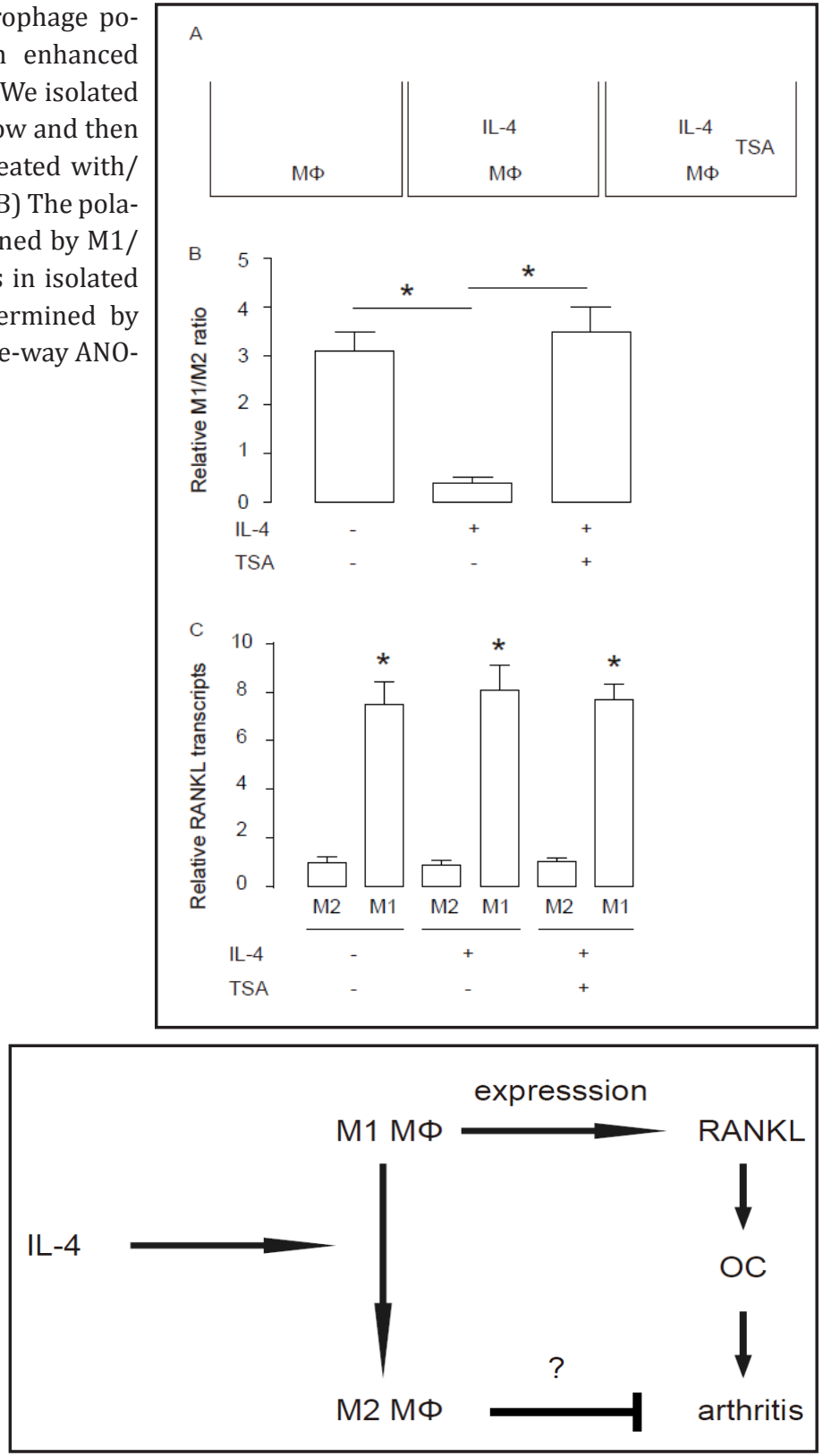

\section{Discussion}

AS is a chronic, high-incident, inflammatory disease that affects the sacroiliac peripheral joints [1-4]. In US and Northern Europe, AS-related disorders have an estimated prevalence of more than 1 in 1000 individuals [1-4]. Disease onset commonly occurs in late adolescence and early adulthood, and nearly $80 \%$ of affected people are symptomatic by the age of 30 [1-4]. In addition to the strong genetic predisposition to express HLA-B27, the characteristic clinical features of AS include inflammatory back pain, usually due to sacroiliitis and enthesitis [1-4]. Although AS typically involves the axial skeleton and the peripheral joints, such as shoulders and hips, extra-articular structures are also affected [1-4].

The etiology and pathogenesis of AS are mainly discerned from studies at autopsy, biopsies of sacroiliac joints and sites of enthesitis [1-4]. Moreover, the effects of IL_4 on AS are not consistently reported in the literature [23, 39-41]. Since the pathology is generally difficult to access, animal models have been widely used to provide opportunities for 
investigating the mechanisms underlying AS molecular pathology [1-4]. There are different types of animal models for AS, including progressive ankyloses model, transgenic model, collagen-induced spondylitis and PGIA [1-4]. Here, we used a well-established PGIA model to study the underlying mechanisms of IL-4-mediated alleviation of arthritis severity.

Our hypothesis is based on studies from other areas [15-25]. IL-4 conducts Th2 lymphocyte-mediated effects [20-25], among which enhancement of M1-to-M2 macrophage polarization has been well described [15-19]. Since macrophages are critical regulators and may even serve as OC precursors [20-25], we hypothesize that regulation of macrophages by IL-4 may be critical for the effects of IL-4 on PGIA.

Here, we injected IL-4 into the articular cavity of PGIA mice. We confirmed the therapeutic effects of IL-4 on PGIA by incidence of arthritis, clinical and pathological arthritis severity and PET tracer uptake. These data validated the model. Then, we isolated macrophages from the articular cavity before and after IL-4 treatment. Interestingly, we did not find changes in the relative percentage of macrophages. These data suggest that IL-4 may not affect the proliferation and/or survival of macrophages. However, IL-4 did significantly polarize M1 to M2 macrophages, based on CD206 positivity [15-19]. And our further analyzes of RANKL levels suggest that RANKL was predominantly produced by M1, but not M2 macrophages. Thus, IL-4 may significantly decrease RANKL levels in macrophage populations. Since RANKL binds to its receptor RANK to activate and differentiate OC [5-7, 20-25], these data may explain the previous findings of significant decreases in OC-mediated bone destruction and loss by IL-4 treatment [5-7]. Hence, the therapeutic effects of IL-4 may be at least partially mediated by inhibition of OC activation and differentiation, through M2polarization of macrophages.

Since these data were obtained from an in vivo mouse model, it is possible that the effects of IL-4 on macrophage polarization are not direct. In other words, IL-4 may need the inflammatory environment to polarize macrophages. Therefore, we examined this possibility in vitro. We not only confirmed that IL-4 independently induces macrophage polarization in vitro, but further demonstrated that the effects of IL-4 on macrophage polarization may involve histone deacetylation, consistent with previous studies on IL-4 in different models [29-33].

Since RANKL activates the NF-kB signaling pathway [5-7], our data suggest that IL-4 may alter histone deacetylation in macrophages to polarize them into an M2 subtype, in which gene expression of RANKL is significantly inhibited, resulting in deactivation of the NF-kB signaling pathway, which is necessary to trigger OC activation and differentiation to mitigate the severity of PGIA or the clinical severity of AS. Future work may analyze OPG levels aside from RANKL in this experimental model [5-7], and may also study the precise molecular events during M1-to-M2 polarization as well as the direct regulation on RANKL gene expression in macrophages [15-19].

Our study sheds new light on the mechanisms underlying the therapeutic effects of IL-4 on AS, and highlights regulation of macrophage polarization as a promising innovative treatment for AS.

\section{Disclosure Statement}

The authors have declared that no competing interests exist.

\section{Reference}

1 Dean LE, Jones GT, MacDonald AG, Downham C, Sturrock RD, Macfarlane GJ: Global prevalence of ankylosing spondylitis. Rheumatology (Oxford) 2014;53:650-657.

2 Golder V, Schachna L: Ankylosing spondylitis: An update. Australian family physician 2013;42:780-784. 
Lin/Qiu/Chen: IL-4 Modulates Macrophages in AS

3 Colbert RA, Tran TM, Layh-Schmitt G: Hla-b27 misfolding and ankylosing spondylitis. Mol Immunol 2014;57:44-51.

4 Robinson PC, Brown MA: Genetics of ankylosing spondylitis. Mol Immunol 2014;57:2-11.

5 Leibbrandt A, Penninger JM: Rankl/rank as key factors for osteoclast development and bone loss in arthropathies. Adv Exp Med Biol 2009;649:100-113.

6 Anandarajah AP, Schwarz EM: Bone loss in the spondyloarthropathies: Role of osteoclast, rankl, rank and opg in the spondyloarthropathies. Adv Exp Med Biol 2009;649:85-99.

7 Tanaka S: Signaling axis in osteoclast biology and therapeutic targeting in the rankl/rank/opg system. Am J Nephrol 2007;27:466-478.

8 Honma M, Ikebuchi Y, Kariya Y, Suzuki H: Regulatory mechanisms of rankl presentation to osteoclast precursors. Curr Osteoporos Rep 2014;12:115-120.

9 Xing L, Schwarz EM, Boyce BF: Osteoclast precursors, rankl/rank, and immunology. Immunol Rev 2005;208:19-29.

10 Takayanagi H, Kim S, Taniguchi T: Signaling crosstalk between rankl and interferons in osteoclast differentiation. Arthritis research 2002;4 Suppl 3:S227-232.

11 Fuller K, Wong B, Fox S, Choi Y, Chambers TJ: Trance is necessary and sufficient for osteoblast-mediated activation of bone resorption in osteoclasts. J Exp Med 1998;188:997-1001.

12 Yasuda H, Shima N, Nakagawa N, Yamaguchi K, Kinosaki M, Mochizuki S, Tomoyasu A, Yano K, Goto M, Murakami A, Tsuda E, Morinaga T, Higashio K, Udagawa N, Takahashi N, Suda T: Osteoclast differentiation factor is a ligand for osteoprotegerin/osteoclastogenesis-inhibitory factor and is identical to trance/rankl. Proc Natl Acad Sci U S A 1998;95:3597-3602.

13 Lacey DL, Timms E, Tan HL, Kelley MJ, Dunstan CR, Burgess T, Elliott R, Colombero A, Elliott G, Scully S, Hsu H, Sullivan J, Hawkins N, Davy E, Capparelli C, Eli A, Qian YX, Kaufman S, Sarosi I, Shalhoub V, Senaldi G, Guo J, Delaney J, Boyle WJ: Osteoprotegerin ligand is a cytokine that regulates osteoclast differentiation and activation. Cell 1998;93:165-176.

14 Davey-Ranasinghe N, Deodhar A: Osteoporosis and vertebral fractures in ankylosing spondylitis. Curr Opin Rheumatol 2013;25:509-516.

15 Gordon S: Alternative activation of macrophages. Nat Rev Immunol 2003;3:23-35.

16 Gordon S, Martinez FO: Alternative activation of macrophages: Mechanism and functions. Immunity 2010;32:593-604.

17 Geissmann F, Manz MG, Jung S, Sieweke MH, Merad M, Ley K: Development of monocytes, macrophages, and dendritic cells. Science 2010;327:656-661.

18 Ricardo SD, van Goor H, Eddy AA: Macrophage diversity in renal injury and repair. J Clin Invest 2008;118:3522-3530.

19 Xiao X, Gaffar I, Guo P, Wiersch J, Fischbach S, Peirish L, Song Z, El-Gohary Y, Prasadan K, Shiota C, Gittes GK: M2 macrophages promote beta-cell proliferation by up-regulation of smad7. Proc Natl Acad Sci U S A 2014;111:E1211-1220.

20 Finnegan A, Grusby MJ, Kaplan CD, O'Neill SK, Eibel H, Koreny T, Czipri M, Mikecz K, Zhang J: Il-4 and il-12 regulate proteoglycan-induced arthritis through stat-dependent mechanisms. J Immunol 2002;169:33453352.

21 Myers LK, Tang B, Stuart JM, Kang AH: The role of il-4 in regulation of murine collagen-induced arthritis. Clin Immunol 2002;102:185-191.

22 Morita Y, Yang J, Gupta R, Shimizu K, Shelden EA, Endres J, Mule JJ, McDonagh KT, Fox DA: Dendritic cells genetically engineered to express il-4 inhibit murine collagen-induced arthritis. J Clin Invest 2001;107:1275-1284.

23 Lubberts E, Joosten LA, Chabaud M, van Den Bersselaar L, Oppers B, Coenen-De Roo CJ, Richards CD, Miossec P, van Den Berg WB: Il-4 gene therapy for collagen arthritis suppresses synovial il-17 and osteoprotegerin ligand and prevents bone erosion. J Clin Invest 2000;105:1697-1710.

24 Cottard V, Mulleman D, Bouille P, Mezzina M, Boissier MC, Bessis N: Adeno-associated virus-mediated delivery of il-4 prevents collagen-induced arthritis. Gene Ther 2000;7:1930-1939.

25 Yoshino S: Treatment with an anti-il-4 monoclonal antibody blocks suppression of collagen-induced arthritis in mice by oral administration of type ii collagen. J Immunol 1998;160:3067-3071. 


\section{Cellular Physiology Cell Physiol Biochem 2015;35:2213-2222 \begin{tabular}{ll|l} 
and Biochemistry & $\begin{array}{l}\text { DOI: 10.1159/000374026 } \\
\text { Published online: April 08, } 2015\end{array}$ & $\begin{array}{l}\text { O 2015 S. Karger AG, Basel } \\
\text { www.karger.com/cpb }\end{array}$ \\
\cline { 2 - 3 }
\end{tabular}}

Lin/Qiu/Chen: IL-4 Modulates Macrophages in AS

26 Veremeyko T, Siddiqui S, Sotnikov I, Yung A, Ponomarev ED: Il-4/il-13-dependent and independent expression of mir-124 and its contribution to $\mathrm{m} 2$ phenotype of monocytic cells in normal conditions and during allergic inflammation. PLoS One 2013;8:e81774.

27 Martinez FO, Helming L, Milde R, Varin A, Melgert BN, Draijer C, Thomas B, Fabbri M, Crawshaw A, Ho LP, Ten Hacken NH, Cobos Jimenez V, Kootstra NA, Hamann J, Greaves DR, Locati M, Mantovani A, Gordon S: Genetic programs expressed in resting and il-4 alternatively activated mouse and human macrophages: Similarities and differences. Blood 2013;121:e57-69.

28 Kuroda E, Ho V, Ruschmann J, Antignano F, Hamilton M, Rauh MJ, Antov A, Flavell RA, Sly LM, Krystal G: Ship represses the generation of il-3-induced $\mathrm{m} 2$ macrophages by inhibiting il-4 production from basophils. J Immunol 2009;183:3652-3660.

29 Lopez-Bravo M, Minguito de la Escalera M, Dominguez PM, Gonzalez-Cintado L, del Fresno C, Martin P, Martinez del Hoyo G, Ardavin C: Il-4 blocks th1-polarizing/inflammatory cytokine gene expression during monocyte-derived dendritic cell differentiation through histone hypoacetylation. J Allergy Clin Immunol 2013;132:1409-1419.

30 Serrat N, Pereira-Lopes S, Comalada M, Lloberas J, Celada A: Deacetylation of c/ebpbeta is required for il-4induced arginase-1 expression in murine macrophages. Eur J Immunol 2012;42:3028-3037.

31 Yamamoto I, Matsunaga T, Sakata K, Nakamura Y, Doi S, Hanmyou F: Histone hyperacetylation plays a role in augmentation of il-4-induced ige production in lps-stimulated murine b-lymphocytes by sodium butyrate. J Biochem 1996;119:1056-1061.

32 Fields PE, Kim ST, Flavell RA: Cutting edge: Changes in histone acetylation at the il-4 and ifn-gamma loci accompany th1/th2 differentiation. J Immunol 2002;169:647-650.

33 Fan X, Roy EM, Murphy TC, Nanes MS, Kim S, Pike JW, Rubin J: Regulation of rankl promoter activity is associated with histone remodeling in murine bone stromal cells. J Cell Biochem 2004;93:807-818.

34 van Meegeren ME, Roosendaal G, Coeleveld K, Nieuwenhuizen L, Mastbergen SC, Lafeber FP: A single intraarticular injection with il-4 plus il-10 ameliorates blood-induced cartilage degeneration in haemophilic mice. Br J Haematol 2013;160:515-520.

35 Lories RJ, Derese I, Luyten FP: Modulation of bone morphogenetic protein signaling inhibits the onset and progression of ankylosing enthesitis. J Clin Invest 2005;115:1571-1579.

36 Braem K, Deroose CM, Luyten FP, Lories RJ: Inhibition of inflammation but not ankylosis by glucocorticoids in mice: Further evidence for the entheseal stress hypothesis. Arthritis Res Ther 2012;14:R59.

37 Weischenfeldt J, Porse B: Bone marrow-derived macrophages (bmm): Isolation and applications. CSH protocols 2008;2008:pdb prot5080.

38 Kaim AH, Weber B, Kurrer MO, Gottschalk J, Von Schulthess GK, Buck A: Autoradiographic quantification of 18f-fdg uptake in experimental soft-tissue abscesses in rats. Radiology 2002;223:446-451.

39 Tarner IH, Nakajima A, Seroogy CM, Ermann J, Levicnik A, Contag CH, Fathman CG: Retroviral gene therapy of collagen-induced arthritis by local delivery of il-4. Clin Immunol 2002;105:304-314.

40 Svensson L, Nandakumar KS, Johansson A, Jansson L, Holmdahl R: Il-4-deficient mice develop less acute but more chronic relapsing collagen-induced arthritis. Eur J Immunol 2002;32:2944-2953.

41 Boyle DL, Nguyen KH, Zhuang S, Shi Y, McCormack JE, Chada S, Firestein GS: Intra-articular il-4 gene therapy in arthritis: Anti-inflammatory effect and enhanced th2activity. Gene Ther 1999;6:1911-1918. 